\title{
A Computer Study of the Orders of Finite Simple Groups
}

\author{
By Edward L. Spitznagel, Jr. and Stephen A. Szygenda
}

In this paper we describe how several results of finite group theory were applied in a computer study of the distribution of the orders of finite simple groups. Besides being of some small interest in themselves, the results obtained here may hopefully give some hint as to what might be proved in general about the distribution of finite simple group orders.

The results used in this study are three in number:

(i) A characterization of the orders of finite simple groups with 2-Sylow subgroups of order $<16$.

(ii) Thompson's characterization of the minimal finite simple groups.

(iii) Sylow's second and third theorems.

Result (i) is stated precisely as follows:

Lemma. Let $G$ be a finite simple (nonabelian) group. Then $|G|_{2} \geqq 4$. If $|G|_{2}=4$, then $G \cong P S L(2, q)$, where $q \equiv 3$ or $5(\bmod 8)$. If $|G|_{2}=8$, then $G \cong P S L(2,8)$ or $P S L(2, q)$, where $q \equiv 7$ or $9(\bmod 16)$, or $J$ (Janko's group), or $|G|=$ $q^{3}(q-1)\left(q^{3}+1\right)$, where $q=3^{2 n+1}$.

Proof. That $|G|_{2} \geqq 4$ is the result of Feit and Thompson [5]. If $|G|_{2}=4$, Theorem 2 of [6] applies. Let $|G|_{2}=8$. There are five nonisomorphic groups of order 8 . One, the quaternion group, is never the 2-Sylow subgroup of a finite simple group [3]. The other nonabelian group of order 8 is dihedral, and if this is the 2Sylow subgroup of $G$, by [6], $G \cong P S L(2, q)$ for $q \equiv 7$ or $9(\bmod 16)$. If the 2Sylow subgroup is abelian, then either $G \cong P S L(2,8)$ or $J$ or else the centralizer of an involution in $G$ is of the form $Z_{2} \times P S L\left(2,3^{2 n+1}\right)$ [9]. In this last case, Janko and Thompson [8] show that $G$ satisfies the hypotheses from which Ward [10] computed the character tables of the Ree groups. Therefore $G$ certainly has the same order as a Ree group.

Result (ii) may be found in [1]. As yet, the proof is unpublished.

These three results were used to test numbers $<10^{11}$. By (i) and (ii), any simple group order which is not the order of a known simple group must be divisible by 48 or by the order of a Suzuki group. Multiples of 48 were tested for divisibility by the order of a minimal simple group. Those divisible were then tested for existence of a prime divisor $p$ for which a $p$-Sylow subgroup must be normal. Similarly, multiples of the orders of the two smallest Suzuki groups were tested by Sylow's theorems.

All natural numbers up to $8,000,000$ were tested, and thereafter, due to limitations in time, 100,000 numbers were tested in each of the intervals $10^{i} \leqq n<2 \times 10^{i}$, $2 \times 10^{i} \leqq n<4 \times 10^{i}, 4 \times 10^{i} \leqq n<7 \times 10^{i}, 7 \times 10^{i} \leqq n<10^{i+1}$, for $i=7,8,9,10$. Each set of 100,000 numbers consisted of ten intervals of 10,000 numbers each, the intervals being chosen at random within the prescribed bounds.

Received September 7, 1967. 


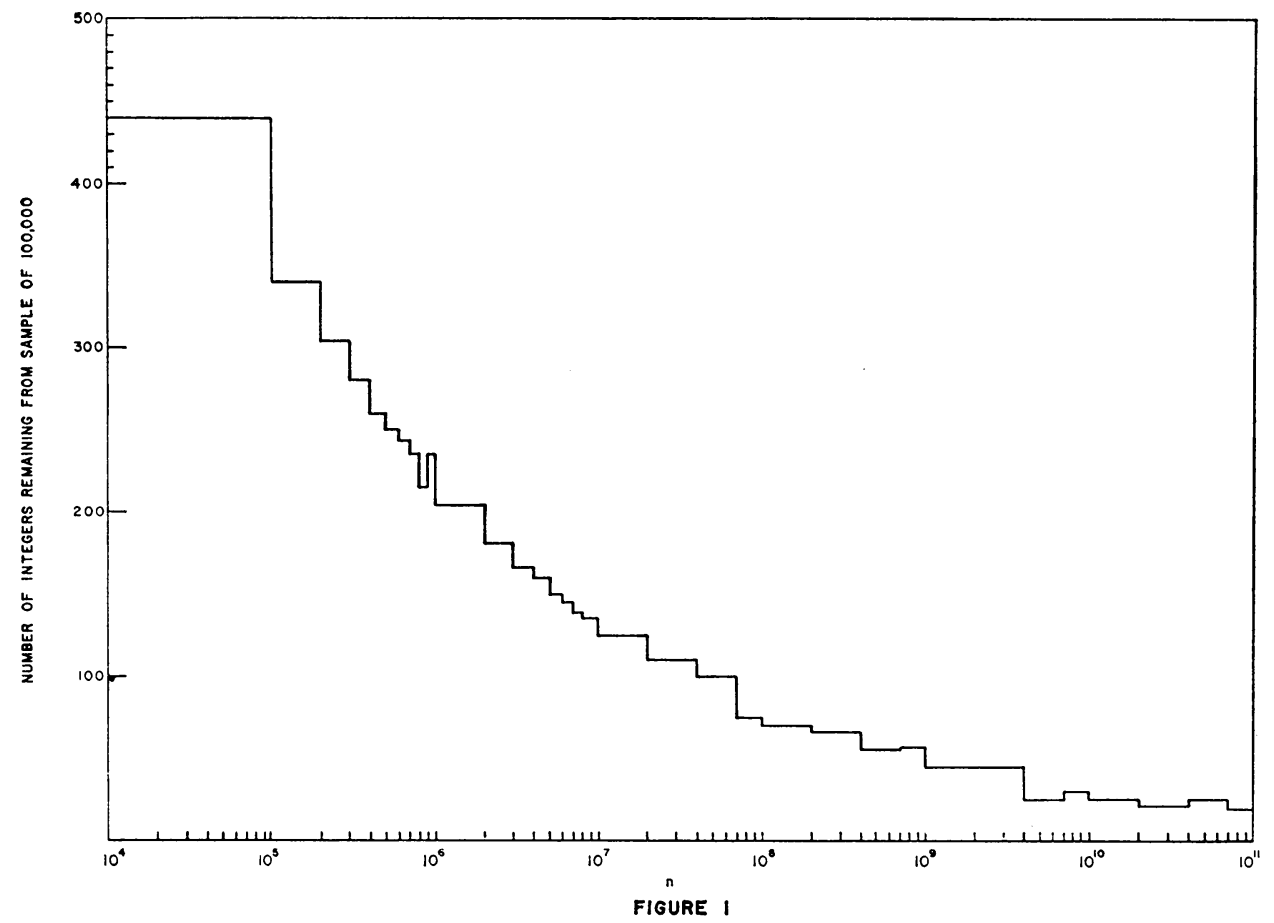

Fig. 1 shows in a histogram the number of integers out of 100,000 which remained as possible orders of simple groups after the eliminations from the tests.

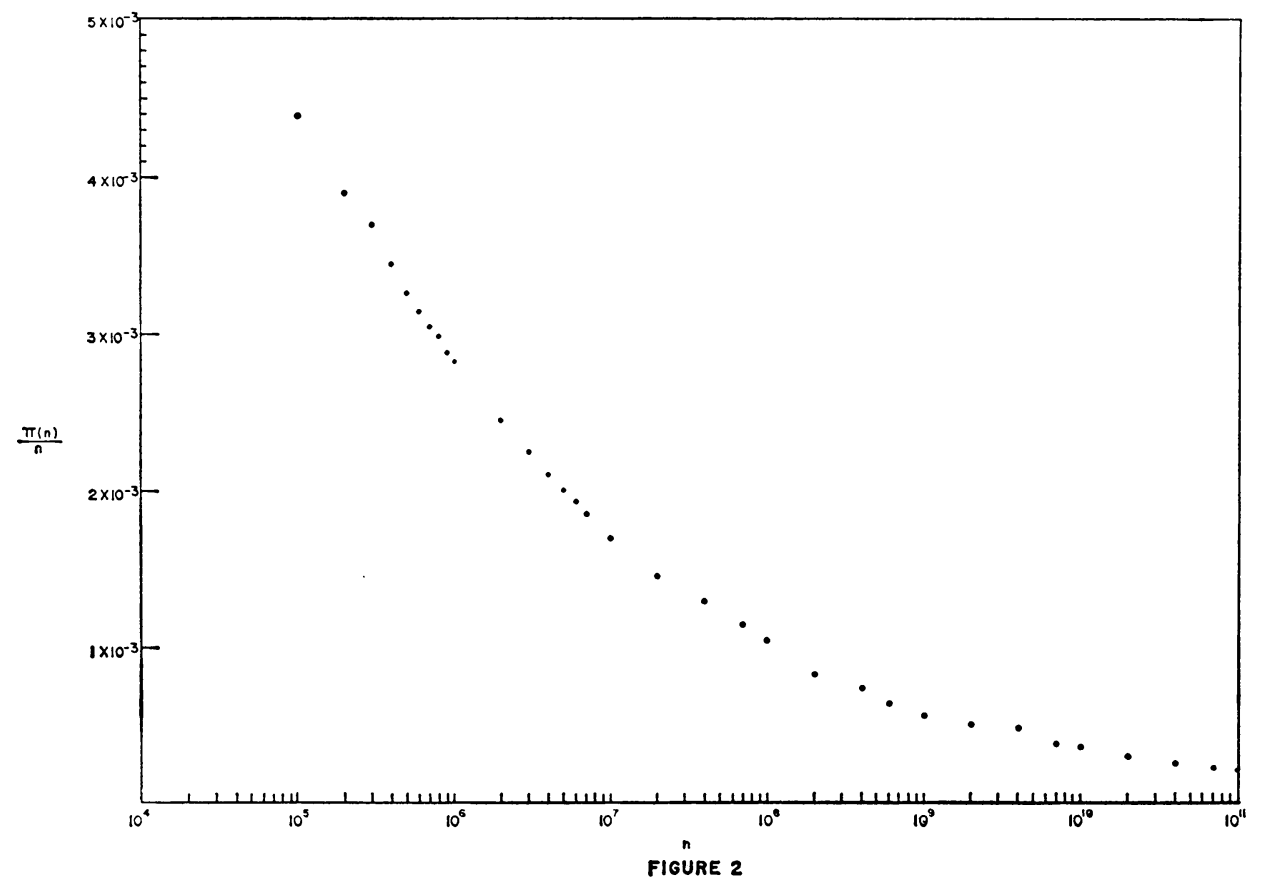


In all likelihood, results about the distribution of simple group orders will be stated in terms of bounds on $\pi(n) / n$, where $\pi(n)$ is defined to be the number of simple group orders less than $n$. If we assume the number of integers left in each of the sets of 100,000 numbers to be proportional to the number which would be left if the entire interval were examined, we can plot certain points along an upper bound for $\pi(n)$. This is done in Fig. 2. From the appearance of the graph, one would guess that $\pi(n) / n \rightarrow 0$ as $n \rightarrow \infty$. This surmise was very recently proved correct [4]. The regular appearance of Fig. 2 gives hope that much more might be proved.

In the course of the work, two other results besides the three listed above were implemented on a trial basis. They were Burnside's transfer theorem [7] and a theorem of Brauer [2]. As neither one produced significant reductions, and each increased the running time tenfold or more, they were not included in the final program. This suggests a question in closing: Are there any other results whose ease of use and effectiveness would be comparable to those used here?

Department of Mathematics

Northwestern University

Evanston, Illinois

1. JA. G. Berkovič, "Finite groups with dispersive second maximal subgroups," Soviet Math. Dokl., v. 5, 1964, pp. 1331-1334. MR 31 \#4836.

2. R. BRACER, "On permutation groups of prime degree and related classes of groups," Ann. of Math. (2), v. 44, 1943, pp. 57-79. MR 4, 266; MR 6, 334.

3. R. BRACER \& M. SUZUKI, "On finite groups of even order whose 2-Sylow group is a quaternion group," Proc. Nat. Acad. Sci. U.S.A., v. 45, 1959, pp. 1757-1759. MR 22 \#31.

4. L. L. Donnhoff, "Simple groups are scarce," Proc. Amer. Math. Soc., v. 19, 1968, pp. $692-696$

5. W. Feit \& J. G. Thompson, "Solvability of groups of odd order," Pacific J. Math., v. 13, 1963, pp. 771-1029. IIR 29 \#3538.

6. D. Gorenstein \& J. H. Walter, "The characterization of finite groups with dihedral Sylow 2-subgroups. I," J. Algebra, v. 2, 1965, pp. 85-162. MR 31 \#1297a.

7. M. HALL, The Theory of Groups, Macmillan, New York, 1959, pp. 203-204. MR 21 \#1996.

8. Z. JANKo \& J. G. THOMPSON, "On a class of finite simple groups of Ree," J. Algebra, v. 4, 1966, pp. 274-293. MR $34 \# 1386$.

9. J. H. WALTER, "The characterization of finite groups with abelian Sylow 2-subgroups." (To appear.)

10. H. N. WARD, "On Ree's series of simple groups," Trans. Amer. Math. Soc., v. 121, 1966, pp. 62-89. MR $33 \# 5752$. 